\title{
Experimental research of plotter printing of HIC thick-film conductors
}

\author{
Anna Zdrok ${ }^{1, *}$, Sergey Artishchev ${ }^{1}$, and Anton Loschilov ${ }^{1}$ \\ ${ }^{1}$ Tomsk State University of Control Systems and Radioelectronics (TUSUR), 634050 Lenina, 40, city \\ Tomsk, Russia
}

\begin{abstract}
The results of complex studies of the plotter printing technology of conductors intended for the manufacture of thick-film HIC are presented. The profile and geometry of the films were investigated. The electrical and mechanical parameters of the films were measured.
\end{abstract}

\section{Introduction: relevance, issues}

Hybrid Integrated Circuit (HIC) can be made using thin-film and thick-film technologies. The difference between such schemes lies in the film deposition method. The choice of the basic technology for circuit manufacturing depends on the range of operating frequencies. Thick-film technology allows to implement elements operating in the frequency range up to $18 \mathrm{GHz}[1,2]$, and it has a wide range of print thicknesses with low resolution (about 70 microns). Thick-film HICs are beneficial at moderately high dissipation powers $(2,5-4,0$ $\mathrm{W} / \mathrm{cm}^{2}$ ). In addition, the use of thick-film technologies in the HIC manufacturing is associated with the simplicity of fulfilling the requirements for design and technological solutions of electronic modules.

Thick-film technology is traditionally based on screen printing. The essence of screen printing is to push the applied material with a squeegee through a mesh screen onto the surface of the workpiece. The use of screen printing is reflected in sheet-to-sheet and rollto-roll printing [3-5].

An alternative to screen printing is non-screen printing or plotter printing. Plotter printing is the process of direct continuous formation (printing) of HIC topology on a dielectric base by dispensing pastes of the required volumes in accordance with the pattern of a given topology [6-7]. In addition to the main advantage (i.e. the absence of special forms used to pattern formation), compared to screen printing, plotter printing allows to provide:

- operative adjustment of the volume of doses of pastes applied;

- the ability to dispense pastes to the bases with side borders;

- preservation of pastes due to the closed container (syringe);

- reduced paste consumption;

- quick start of production due to program preparation of templates and setting the equipment.

\footnotetext{
*Corresponding author: kundep@mail.ru
} 
However, the plotter printing technology until recently was incomplete and had the following disadvantages:

- lack of universal algorithms for adapting technological equipment for manufacturing different products;

- models of dependence of dosed materials behavior on the conditions of exposure to dosing mechanisms and environmental parameters have not been studied.

In this paper, we studied the technology of layer-by-layer growth of the HIC structure by the method of printer-based dosing of functional materials (silver-containing pastes) and tried to develop some algorithm of work for further improvement of the proposed thick-film technology for the manufacturing of the HIC elements and products.

\section{Objects of research and experimental technique}

\subsection{Description of technology: operating principle, equipment, materials}

\subsubsection{The principle of dosing and formation of prints}

The purpose of the plotter printing process is to apply a predetermined amount of paste on the substrate in accordance with the template of the required topology with a certain accuracy and controlled repeatability.

The pattern is formed by continuous vector lines. This allows us to ensure surface smoothness and evenness of borders $[8,9]$ in comparison with raster imaging techniques. It should also be noted that if it is necessary to create a polygon of a certain area, the merging of extended lines also provides the best surface topography compared to the point overlap of individual drops.

The quality of interaction of the dosed material with the substrate surface is determined by the surface wettability. The wettability, in turn, is determined by the chemical composition of the materials, as well as the quality of treatment of the substrate surface.

Directly dosing of material is carried out from a closed container like a syringe. A dispensing needle is attached to the dispensing hole. The diameter of the needle hole determines the base value of the diameter / width of the print (the print value varies slightly depending on other factors). Also, the internal shape of the needle is responsible for the movement of material from the container dispensing hole to the final dispensing hole of the needle. The movement of material in the tank is carried out due to mechanical impact of pneumatic, piston or screw type.

In this paper, the piston-type dosing method is considered. The piston exerts pressure on the mass of material in the tank, moving with a given step in the vertical direction. The choice of this method is based on the simplicity of the design of the metering device, as well as the accuracy of adjusting the pressure on the column of material in the tank.

\subsubsection{Plotter printing plant design, experimental equipment}

Within the framework of research, in addition to adjusting of the technological process, a plotter printing installation is being developed (a model is presented in [6]). However, in this work, at the stage of preliminary tests designed to assess the limits and limitations of the piston dosing method, an analog of our device, the 3D printer Voltera V-One (Voltera), was used as experimental equipment (Fig. 1) [10]. The diameter of the nozzle of the outlet is $200 \mu \mathrm{m}$. 


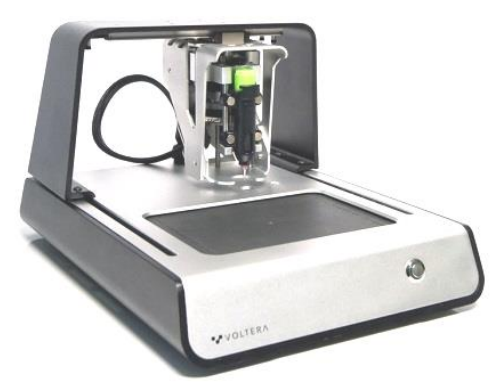

Fig. 1. Appearance of the Voltera V-One Printer.

\subsubsection{Experimental materials}

Pastes and substrates were used as experimental materials for studying the plotter printing of thick-film HIC conductors.

The substrate material is ceramic (Al2O3). Ceramic substrates are currently one of the main materials for creating HIC. The advantages of vacuum-tight corundum-based ceramics include: resistance to various acids, alkalis and other aggressive environments, the presence of excellent strong insulation properties, as well as resistance to sudden temperature changes. In this work, studies were carried out on substrates of the VK-96 brand. Substrate size: $60 \times 48 \times 0.5 \mathrm{~mm}$.

Conductive paste Conductive Ink, Voltera (Canada) (technical characteristics in Table 1) became the material for dosing and forming metal prints. Generally the choice of printed material determined by the limitations of the dosing device.

Table 1. Technical specifications of Conductive Ink Voltera.

\begin{tabular}{|l|l|}
\hline \multicolumn{1}{|c|}{ Parameter Name } & \multicolumn{1}{c|}{ Parameter Value } \\
\hline Appearance & $\begin{array}{l}\text { Viscous homogeneous mass of } \\
\text { brown color }\end{array}$ \\
\hline Conducting phase material & Silver \\
\hline Organic binder base & 1 -methyl-2-pyrrolidone, phenol \\
\hline Kinetic viscosity, $\mathrm{mm} 2 / \mathrm{s}$ & $>40\left(23^{\circ} \mathrm{C}\right)$ \\
& $>20,5\left(40^{\circ} \mathrm{C}\right)$ \\
\hline Surface resistivity at a thickness of $50 \mu \mathrm{m}, \mathrm{mOhm} / \mathrm{sq}$. & 20 \\
\hline
\end{tabular}

\subsection{Experimental technique}

The experimental research algorithm is based on the technological route of manufacturing a simplified HIC element, which includes:

1) development of the topology of the experimental sample;

2) processing and preparing the surface of the substrate for applying pastes;

3) applying pastes to the prepared substrate;

4) heat treatment of the substrate with a deposited layer of pastes;

5) testing of samples and processing of results.

\subsubsection{Topology of the experimental sample}

The topology was designed in accordance with the following requirements:

1) to assess the geometric and electrical parameters of the films - a single line; 
2) to assess the geometric, electrical, mechanical parameters of the films - a polygon consisting of several single lines.

Based on the requirements, it was decided to use two types of topologies:

1) Topology №1 (Fig. 2) contains:

- block of lines located in 2 columns of 16 pieces: line length $10 \mathrm{~mm}$, estimated line width $0.2 \mathrm{~mm}$, spacing between lines $5 \mathrm{~mm}$, spacing between columns $5 \mathrm{~mm}$;

- a block of polygons designed to measure mechanical parameters, size $5 \times 5 \mathrm{~mm}$, arranged in 3 columns of 6 pieces: the interval between polygons $5 \mathrm{~mm}$, the interval between columns $5 \mathrm{~mm}$;

2) Topology №2 contains a block of polygons (Fig. 3), arranged in 3 columns of 6 pieces, designed to measure surface electrical resistance by the Van der Pauw method.
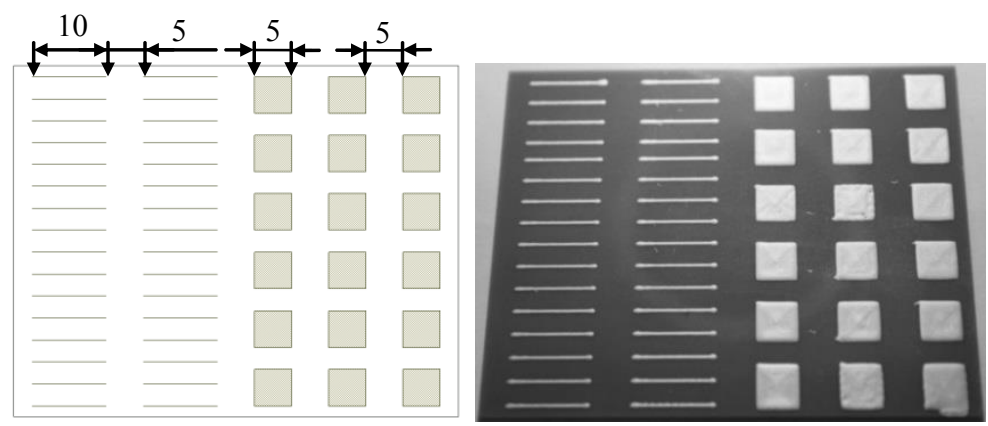

Fig. 2. Experimental sample No. 1: topology (left) and photograph (right).
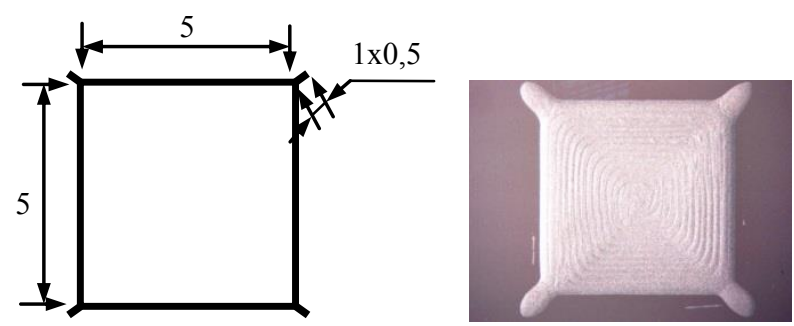

Fig. 3. Experimental sample No. 2: the geometry of the picture (left) and the photograph (right).

\subsubsection{Modes of heat treatment of experimental samples}

After printing of conductive materials, the substrates were heat treated in a PM-1700p muffle furnace. This operation is designed to evaporate a binder and sinter particles of silver. The samples were processed at four different temperatures in order to reveal the influence of the heat treatment parameters on the electrical and mechanical parameters of the films. Figure 4 shows the thermal profiles.

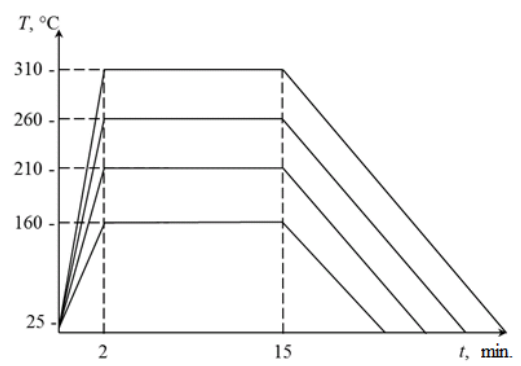

Fig. 4. Thermal profiles of post-processing of experimental samples. 


\section{Experimental research}

After heat treatment, experimental measurements of the parameters were carried out, which included:

1) measurement of film thicknesses (equipment used: KLA Tencor profilometer);

2) assessment of the geometry and surface structure of the films (equipment used: KLA Tencor profilometer);

3) measurement of the electrical resistance of the films (equipment used: immitance meter E7-20);

4) measurement of adhesion (equipment used: test bench dynamometric MEGEON 03000D);

5) statistical data processing.

\subsection{Investigation of the surface structure, geometry and profile of films}

The shape and structure of the films significantly affect the quality of signal transmission along the line. When evaluating films obtained by plotter printing method, the following parameters were considered:

- width, length and thickness of the films, their uniformity over the entire surface, the deviation from the reference value of output hole diameter;

- film profile, sharpness of boundaries;

- surface roughness (the assessment was carried out according to profilograms, the value is the arithmetic average of the absolute values of the deviations of the profile within the base length of the profile peak).

Figure 5 shows photographs of film-lines at a magnification scale of $45 \mathrm{X}$, and figure 6 shows examples of profilograms.

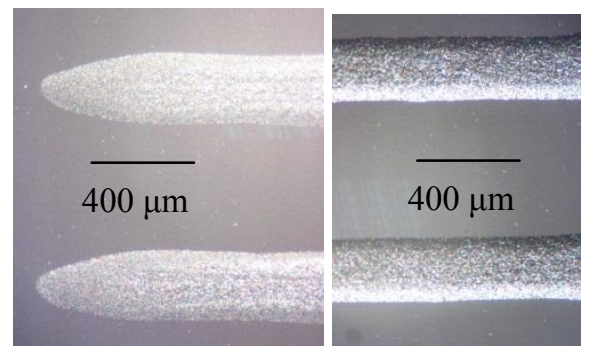

Fig. 5. Photo of single line films on the scale of $45 \mathrm{X}$.

The following results are obtained:

1) width $-400 \pm 50$ microns; length $-1000 \pm 50$ microns; thickness $-18 \pm 2$ microns;

2) shrinkage of the thickness with increasing sintering temperature: about $20-22 \%$;

3) flowing of paste: about $100-125 \%$ of the base value of the outlet diameter;

4) uneven film shape, slight thickening at the ends of the lines (Fig. 5, left);

5) profile change with increasing sintering temperature: from drop-shaped (triangular) to rectangular (Fig. 6);

6) the clarity of the borders: even edges in length;

7) surface roughness (Ra): from 1.5 to 2 microns with increasing temperature.

Figure 7 shows a photograph of a film-polygon at a magnification scale of $45 \mathrm{X}$, and figure 8 shows examples of profilograms. 


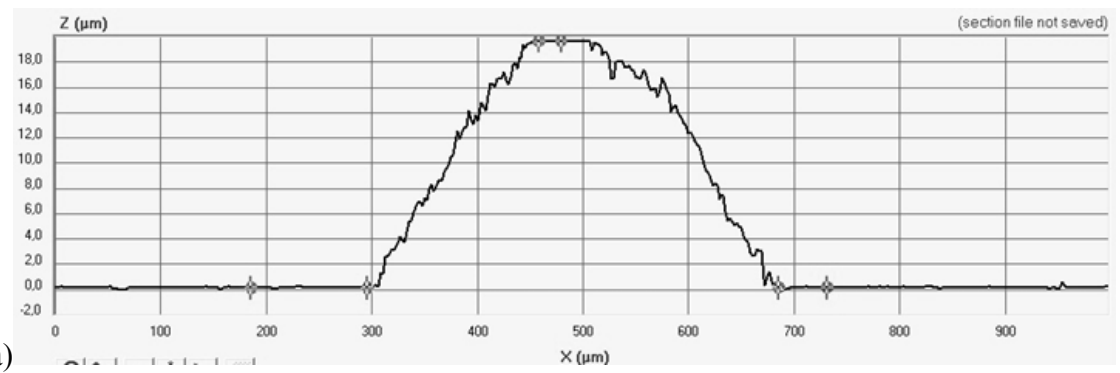

a)

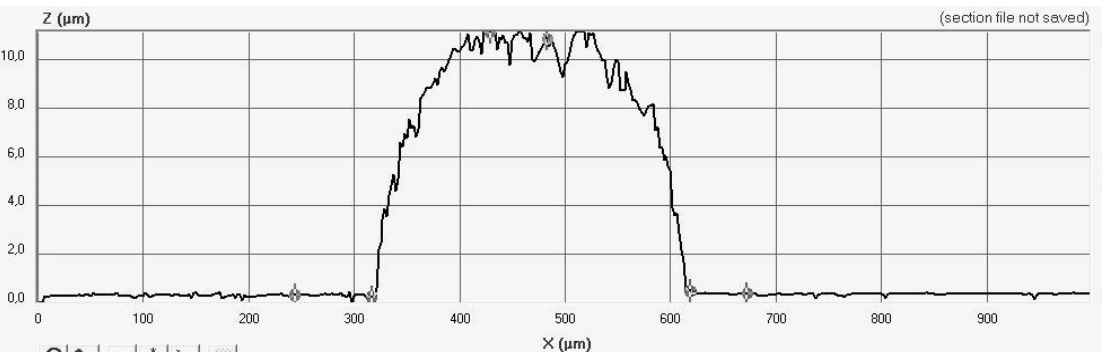

Fig. 6. Examples of profilograms of films after heat treatment: a) $180^{\circ} \mathrm{C}$, b) $310^{\circ} \mathrm{C}$.

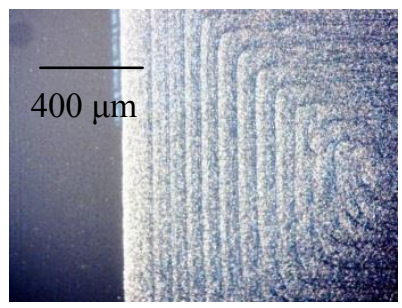

Fig. 7. Photo of film-polygon on scale of $45 \mathrm{H}$.

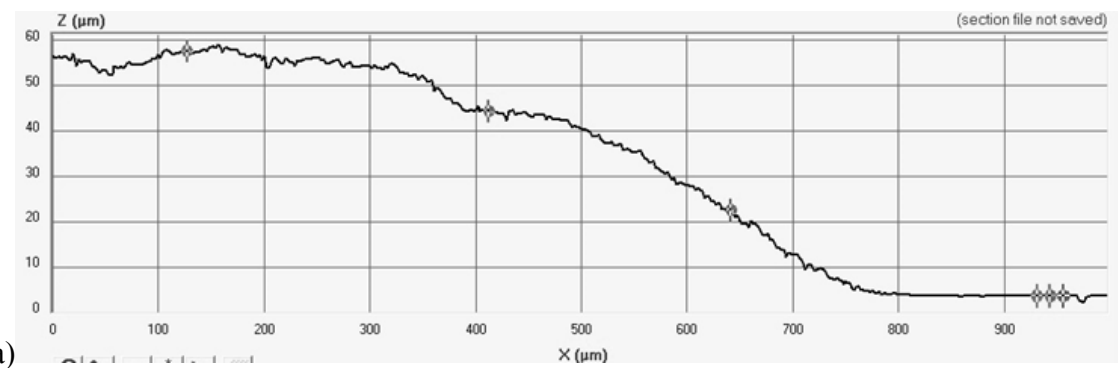

a)

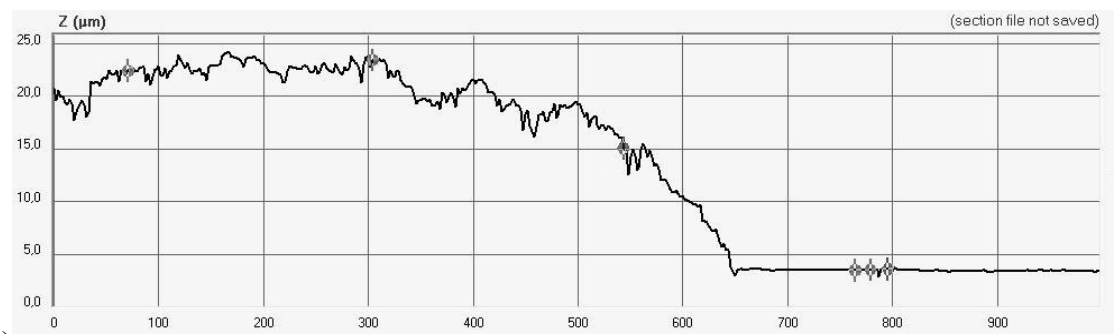

b)

Fig. 8. Examples of profilograms of films after heat treatment: a) $180^{\circ} \mathrm{C}$, b) $310^{\circ} \mathrm{C}$. 
The following results are obtained:

1) width $-5000 \pm 50$ microns; length $-5000 \pm 50$ microns; thickness $-55 \pm 5$ microns;

2) shrinkage of the thickness with increasing sintering temperature: about $25-30 \%$;

3) flowing paste: about $50-75 \%$ along the edge of the line; path;

4) profile: unevenness is observed when the lines overlap in accordance with the nozzle

5) border: with increasing temperature, the film acquires more rectangular clear edges;

6) surface roughness $(\mathrm{Ra})$ : from 5 to 10 microns with increasing temperature.

\subsection{The research of electrical and mechanical parameters of films}

After measuring the parameters, statistical processing of the data was carried out, according to the results of which a dependence of the arithmetic mean value of the surface electrical resistance on the annealing temperature of film-lines (Fig. 9) was obtained. For filmpolygon, the value of electrical surface resistance remained unchanged: about $192 \mathrm{kOhm} / \square$.

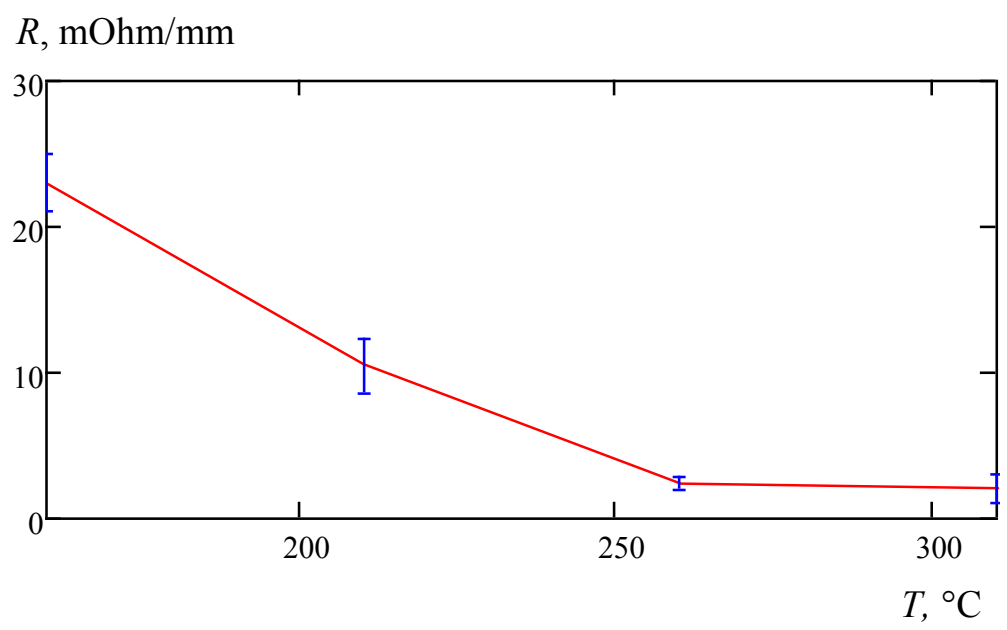

Fig. 9. Dependence of surface electrical resistance on the annealing temperature of film-line samples.

The mechanical parameter of the films is adhesion. The adhesion was measured by measuring the force of direct separation of the films from the substrate. A pin was attached to the film by soldering (the cross-sectional area of the pin was equal to the surface area of the film). The pin was attached to the adhesion meter by means of a rigid screw connection (see point 3 ). The separation was carried out in a strictly perpendicular direction of the force to the film surface. According to the results of the measurements, a wide scatter of values is observed. This phenomenon is supposedly due to the instability of the material during repeated temperature exposure and low solderability.

\section{The discussion of the results}

During experimental studies for the material Conductive Ink, Voltera (Canada) and for the piston method of dispensing (device 3D printer Voltera V-One) the following was obtained:

1) with increasing sintering temperature:

- the value of the film thickness decreases from 18 to 12 microns for single lines, and from 60 to 30 microns for polygons; 
- the geometry and profile of the films changes - due to an increase in temperature, the most efficient evaporation of the binder and sintering of the metal particles occurs, the film takes on the most rectangular shapes;

- with an increase in sintering temperature over $250{ }^{\circ} \mathrm{C}$, the conductivity increases $4-5$ times: from 25 to $5 \mathrm{mOhm} / \square$;

- the adhesive strength of the materials is reduced (peeling of the material at high temperatures, Fig. 11);

2) there is no possibility of using more viscous materials, insufficient pressure force of the piston;

3) the high wettability of the substrate material used in the tasks of manufacturing microwave HIC (violations in the geometry and profile of the films, Fig. 11);

4) the impossibility of regulating print resolution.

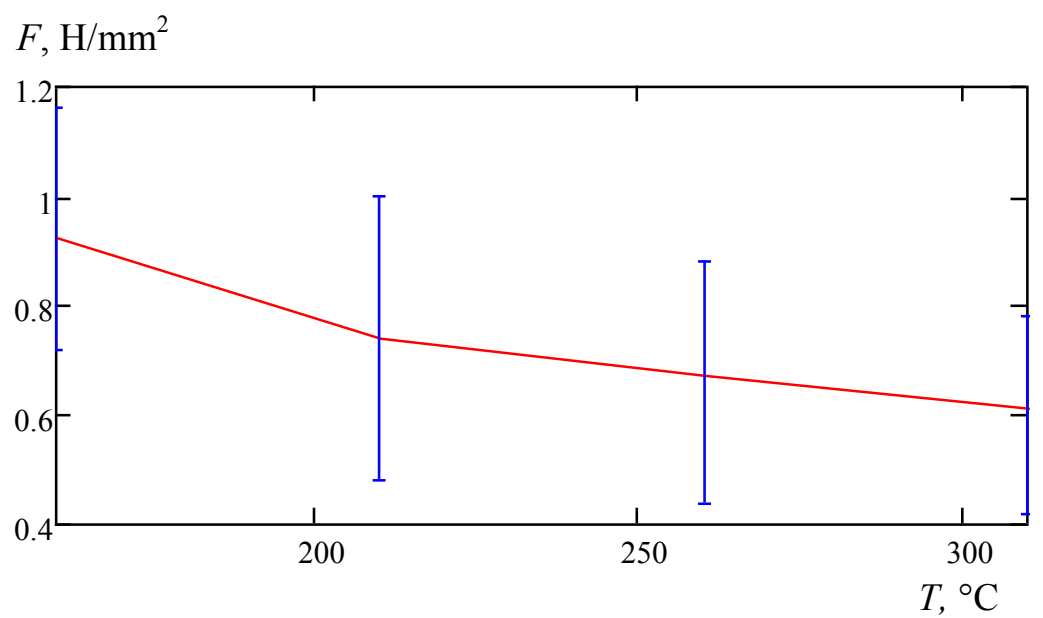

Fig. 10. Dependence of the separation force on the annealing temperature of samples.

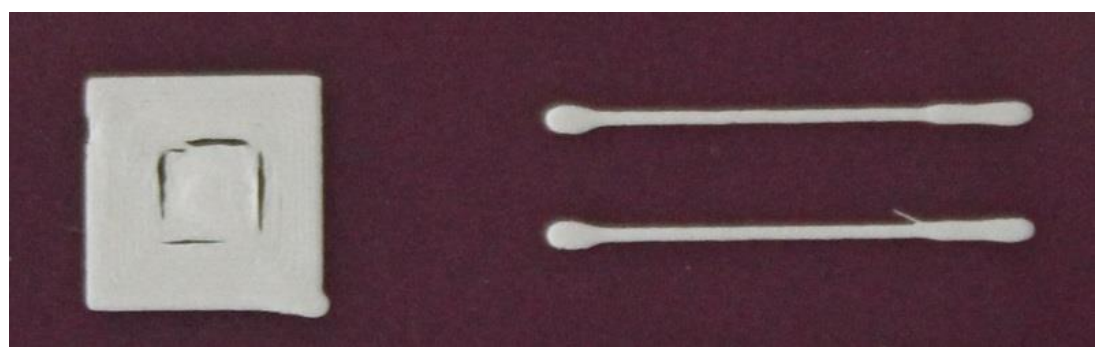

Fig. 11. Photograph of deformation and heterogeneity of films.

The main results should be attributed:

1) the considered method of dispensing materials allows to obtain films with a thickness of 15 microns for a single line and 55 microns for a polygon, while ensuring high conductivity (electrical resistance from $20 \mathrm{mOhm}$ );

2) a significant influence on the characteristics of the films is exerted by the choice of the temperature profile of post-processing (annealing of printed structures);

3) control and regulation of the film profile is directly related to the chemical composition of the pastes, which determines:

- viscosity of materials and surface wettability - thickness of prints, spreadability of material (increasing the viscosity of materials will require changes to the dosing system); 
- thixotropy of a substance - a change in the viscosity of a material under mechanical impact (it is necessary to simulate the nature of the material behavior depending on the method of mechanical impact);

- the adhesion of the film and the substrate - to increase the adhesive strength of the material in the composition the presence of substances that contribute to the formation of a special sub-layer during annealing of the sample is required;

4) the dosing system determines the print quality and resolution of the technology; when adjusting the process, it is necessary to take into account the gap between the nozzle and the substrate, and the piston pressure force.

The reported study was funded by RFBR according to the research project № 18-38-00664.

\section{References}

1. Barbara Dziurdzia, Improved Screen Printing for Microwave Hybrids. Limits and Prospects, XXIII IMAPS Poland Conference, pp. 217-222 (1999)

2. M.M. Filyak, Structural and technological foundations of microelectronics, Federal State Budgetary Educational Institution of Higher Education «Orenburg State University», P. 112 (2011)

3. Salam B., Shan X. C., Wei Jun, Large Area Roll-to-Roll Printing of Electrically Conductive Circutries, IEEE $18^{\text {th }}$ Electronics Packaging Technology Conference (EPTC), pp. 250-253 (2016)

4. Frieder Lucklum, Gerrit Dumstorff, 3D Printed Pressure Sensor with Screen-Printed Resistive Read-Out, IEEE Sensors (2016)

5. Saleem Khan, Leandro Lorenzelli, Ravinder Dahiya, Technologies for Printing Sensors and Electronics over Large Flexible Substrates: A Review, IEEE Sensors Journal, pp. 1-22 (2014)

6. Zdrok A.E., Sherstyuk D.V., Umarov A.M., Artishchev S.A., Loschilov A.G., Malyutin N.D. A microstrip line made by plotter printing with ink containing silver nanoparticles. Its frequency and impulse characteristics, Nanotechnology: development and applications - XXI Century, 3(8), pp. 3-8 (2016)

7. Artishchev S.A., Zdrok A.E., Loschilov A.G., Malyutin N.D., Analysis of influence annealing parameters on the electrical resistance of printed electronics, 24th International Crimean Conference Microwave \& Telecommunication Technology, 1, pp. 690-691 (2014)

8. Allanurov A.M., Zdrok A.Ye., Loschilov A.G., Malyutin N.D., Problem of Ink Evaporation while using Plotter Systems to Manufacture Printed Electronic Products, Procedia Technology, v. 18., pp. 19-24 (2014)

9. Zdrok A.E., Malutin N.D., Loschilov A.G., Tuev V.I., The Technology to Form an Organic and Inorganic Material Film by Ultrasonic Capillary Printing, IV Forum Strategic Partnership of Universities and Enterprises of Hi-Tech Branches (Science. Education. Innovations), pp. 109-111 (2015)

10. VOLTERA, Website: https://www.voltera.io (2019) 